\title{
Interactive comment on "Socio-Hydrologic Modeling of the Dynamics of Cooperation in the Transboundary Lancang-Mekong River" by You Lu et al.
}

You Lu et al.

fq.tian@gmail.com

Received and published: 23 November 2020

Reviewer\#1:

Reviewer comment: General comments The authors present a socio-hydrological model of cooperation in transboundary watersheds, using the Lancang-Mekong river basin as a case study. In the model (and case study), upstream countries seek to develop the river for hydropower generation whereas downstream countries rely on river flow for agriculture and fisheries. Cooperation in the model is realized when upstream riparian countries adjust reservoir operations and forgo economic gains for the benefit of downstream riparian countries. This cooperation occurs in response to "coopera-

Printer-friendly version

Discussion paper 
tion demand" of downstream countries, which increases when ecosystem services decrease in these countries. The essential feedback within the model (i.e., cooperation) also depends on the disposition of the upstream riparian to their downstream partners, broadly representing the geopolitical relationship between countries. Cleverly, reservoir operation varies between the ideal operation for upstream countries (optimize hydropower) and downstream countries (e.g., optimize agricultural production), which allows cooperation to be quantified as a value between 0 and 1 . The manuscript focuses on model development and validation rather than hypothesis testing. The novelty of the manuscript therefore rests primarily in the quantitative formulation of transboundary cooperation, including a novel and parsimonious representation of transboundary politics and decision making. This question is of great concern to understanding longterm streamflow trajectories in transboundary basins. The hydrological portion of the model is rigorously validated, and the economics portion of the model is based on established models. The cooperative and political components of the model are also based on published literature. However, given the novelty of this aspect of the model and importance to the nonlinearity / feedback mechanism within the model, the cooperative aspects merit additional consideration within the manuscript, as I describe in "Specific comments".

Author response: We would like to thank reviewer \#1 for the constructive suggestions and comments, which we believe will help to improve the manuscript substantially. We agree to address the main issues raised by the reviewer, and our explanations and responses to all the reviewer's comments and questions are listed as follow.

Reviewer comment: Overall the manuscript is clearly written and presents an important and novel contribution to better understand and model cooperation in transboundary basins. My primary concern relates to parameter selection and calibration, especially those parameters pertaining to cooperation (as I describe below). Specific comments Although the cooperation portion of the model appears to be designed in a manner that is consistent with cooperation in the Mekong, parameterization and validation of

Printer-friendly version

Discussion paper 
this component of the model is given relatively little attention. This raises the question of whether or not the (geo)politics represented in the model (specifically, variables $\mathrm{P}, \mathrm{s}$, and $\mathrm{B} \_\mathrm{h}, \mathrm{FC}$ ) can be parameterized a priori, or if they need to be calibrated a posteriori to cooperation. The method of selecting these variables should be more clearly described within the manuscript (beyond a statement of their values on lines 609-613). This information is essential to understand how the model could be used to understand transboundary cooperation beyond the Mekong, and along those lines it would be helpful for the authors to more clearly detail how they envision future applications of the model would support (and help understand) transboundary cooperation in other regions. This would also give more weight to the statement (abstract, lines 42-44) that "the socio-hydrological model used here provides a useful new framework to investigate and improve transboundary water management elsewhere."

Author response: We appreciate this suggestion on the parameterization and validation of this model, which bring us to realize that the present introduction of parameterization is insufficient. Generally, the model aims to simulate the cooperation evolution in Lancang-Mekong River, which is a typical transboundary river where the upstream benefit from hydropower generation and the downstream gains irrigation and fishery products. Therefore, the model framework can be extended to many other transboundary rivers with dams in the upstream and agriculture and/or fishery in the downstream, when the input data and parameters are well adjusted. Critical parameters in the model are classified into two groups, i.e., parameters in benefit calculation module and parameters in policy feedback module. The parameters in benefit calculation module are extracted from literature or calibrated against the statistical benefits, which will be explained specifically in the next paragraph. The parameters in policy feedback module are set according to the reality of Lancang-Mekong cooperation and then adjusted based on the simulations of cooperation demand and cooperation level, which is still rough because there are limited research and knowledge on the quantification of cooperation and political benefits. Furthermore, as one result of another related paper we are still working on, the long-term sensitivity analysis of the parameters in our model

Printer-friendly version

Discussion paper 
show that the simulation results are more sensitive to parameters in benefit calculation module, including price of rice pa, price of fishery pf, electricity price ph, but not sensitive to parameters in policy feedback module, such as sensitivity of agriculture loss and fishery loss $\varepsilon_{-}$a and $\varepsilon_{-} f$, responsive change rate $s$ and the shape parameter $\beta$. Since the parameters in benefit calculation module are reliable, the uncertainty of simulation is limited and the simulations are credible. When the model is applied in other similar transboundary rivers, the parameters in benefit calculation module need to be extracted from literature such as price data, or calibrated against statistical data of sector benefits, which will ensure the reliability of benefit calculation parameters. As for the parameters in policy feedback module, such as political factor $P$, responsive change rate $S$, and sensitivity of agriculture loss and fishery loss $\varepsilon_{-} a$ and $\varepsilon_{-} f$, they should be "calibrated" so that the simulated cooperation demand and cooperation level are consistent with reality and sentiment analysis data. When the model is applied in enormous cases, these policy feedback parameters could be investigated to find some patterns, which could be then used to determine the corresponding parameters a priori when apply to a new case. Specifically, the parameterization in Lancang-Mekong River is explained as follows. In the benefit calculation module, the price of rice pa in line 382 and the price of fishery pf in line 403 are extracted from MRC (2018). In the policy feedback module, the sensitivity of agriculture loss and fishery loss $\varepsilon$ a and $\varepsilon \_f$ in line 432 are assigned equally as 0.5 in this study, indicating the same importance of the two sectors. The assignments of responsive change rate $s$ and political factor $P$ are introduced in lines 605-611, and we will move the introduction of these two parameters forward to Section 3.4. As for the variables B_(h,FC) and B_(h,NC) mentioned by the reviewer, they indicate the hydropower benefits of upstream countries under altruistic scenario and self-interested scenario respectively, which are explained in 449-453. They are both calculated based on the equation (2) in line 344, in which the electricity price ph is extracted from MRC (2018), and the hydropower generation efficiency $\eta$ is calibrated against the annual power generation data (Yu et al. 2019). The monthly release $Q \_r$ and water head difference $\Delta h$ are calculated under altruistic

Printer-friendly version

Discussion paper 
scenario and self-interested scenario respectively in reservoir operation module. We will add the illustrations above to the manuscript to make the introduction of parameterization clearer.

Reviewer comment: The dynamics of cooperation within the Mekong have been described qualitatively in other papers referenced in the manuscript. Indeed, these papers give confidence to the model as the authors demonstrate that the model is consistent with this qualitative narrative, both in terms of formulation and outcome. However, this raises the question of how the model generates additional insights about cooperation in the Mekong basin beyond what has already been described elsewhere. This particular aspect should be clarified within the manuscript, i.e., what specific insights about transboundary cooperation has the model generated that were not apparent from previous research? An additional aspect of the manuscript that was novel was the use of Lexis-Nexis sentiment analysis, and I suggest this aspect be given more attention in the introduction (at present, it seems downplayed).

Author response: we thank the question raised by the reviewer. As discussed in the introduction and discussion part, the model is the first one to include the evolutionary dynamics of cooperation driven by hydrological variability and human activity as an internal variable. It enables the mid- and long-term evolutionary analysis of transboundary river cooperation and its driven mechanism, which is the unique insight this model can offer. Besides, the model is also a useful tool to analyze the impacts of hydrological, economic and political factors on transboundary river cooperation and project the evolution in the future. We have gained some insights in future projection based on the model, which will be explained in another paper under preparation. For short, new results include that under RCP6.0 emission scenario, the risk of conflict will not increase significantly in the basin, the downstream irrigation benefit will increase by $30 \%$ and Laos hydropower generation will increase by $120 \%$. Both irrigation expansion and runoff decrease will lead to higher cooperation demand, and when the runoff under RCP6.0 decrease by $30 \%$ and irrigation expand as planned, reservoir construction and 
operation of upstream will exceed increasing weight on political benefit of upstream in reducing conflict risks. But in this paper, development of the model is the main work of this manuscript, which has its own novelty and significance. We would like to highlight the distinctions from other model research and new insights the model can offer in the introduction part. Also, we agree that the use of sentiment analysis is a novelty of this manuscript. But we focus on sentiment analysis in another paper submitted to the same special issue on HESS (https://hess.copernicus.org/preprints/hess-2020390/hess-2020-390.pdf), and we would like to reinforce the introduction of the sentiment analysis rather than focus on it in this manuscript.

Reviewer comment: Technical corrections The second sentence of the abstract seems informal and lacks precision, and I suggest replacing "etc." with either additional examples or more precise phrasing.

Author response: We appreciate the suggestion. We will replace "etc." with navigation and ecological services.

Reviewer comment: Parameter selection would be more appropriate in the model development section, opposed to the results section (e.g., lines 609-613).

Author response: We appreciate the suggestion. We will move this part forward to Section 3.4.

Reviewer comment: Lines 625 - 638 would fit better in the Model section, perhaps as a validation subsection.

Author response: We appreciate the suggestion. We will introduce the sentiment analysis after Section 3.4 and remain the comparison between sentiment analysis result and simulated cooperation demand as it be.

Printer-friendly version

Reviewer comment: Subsections in Section 2 are numbered incorrectly as 3.1, 3.2, etc (should be 2.1, 2.2, etc).

Discussion paper

Author response: We appreciate the suggestion. We will correct them. 
Reviewer comment: Using "iff" as a variable name is confusing, because in mathematics notation it means "if and only if" (line 402)

Author response: We appreciate the comment. We will change iff to other expression.

Reviewer comment: On the first read it was a bit confusing to have two equivalent variables for cooperation, delta2 and $\mathrm{C}$. Perhaps it would be helpful to include a note in figure 3 that the two are equal.

Author response: We appreciate the comment. We will add this equation in figure 3.

\section{Reference:}

MRC (2018). Summary State of the Basin Report 2018, Mekong River Commission.

Yu Y., Zhao J., Li D., et al. (2019). Effects of Hydrologic Conditions and Reservoir Operation on Transboundary Cooperation in the Lancang-Mekong River Basin. Journal of Water Resources Planning and Management 145(6): 04019020.

Interactive comment on Hydrol. Earth Syst. Sci. Discuss., https://doi.org/10.5194/hess-2020$388,2020$. 